\title{
Estimation of Physiologic Ability and Surgical Stress Scoring System Appraises Laparoscopy-Assisted and Open Distal Gastrectomy in Treatment of Early Gastric Cancer ${ }^{*}$
}

\author{
Hideki Bou ${ }^{1 \#}$, Hideyuki Suzuki ${ }^{1}$, Kentaro Maejima ${ }^{1}$, Hidetsugu Hanawa ${ }^{1}$, Masanori Watanabe ${ }^{1}$, \\ Eiji Uchida ${ }^{2}$ \\ ${ }^{1}$ Institute of Gastroenterology, Nippon Medical School Musashi-kosugi Hospital, Kawasaki, Japan; ${ }^{2}$ Department of Surgery, Nippon \\ Medical School, Tokyo, Japan. \\ Email: "bou@nms.ac.jp
}

Received July $24^{\text {th }}, 2013$; revised August $23^{\text {rd }}, 2013$; accepted August $30^{\text {th }}, 2013$

Copyright (C) 2013 Hideki Bou et al. This is an open access article distributed under the Creative Commons Attribution License, which permits unrestricted use, distribution, and reproduction in any medium, provided the original work is properly cited.

\begin{abstract}
Laparoscopy-assisted distal gastrectomy (LADG) has been widely used to treat early gastric cancer (EGC). The Estimation of Physiologic Ability and Surgical Stress (E-PASS) scoring system predicts the risk of fatal postoperative complications by quantifying the patient's reserve and degree of surgical stress, but there have been a few reports of use of the E-PASS scoring system to assess the risk of mortality following special types of surgical procedures such as LADG. In this study we assessed the feasibility of LADG versus open distal gastrectomy (ODG) by the E-PASS scoring system. The subjects of this study consisted of 69 stage IA gastric cancer patients who underwent LADG (LADG group) and 69 stage IA gastric cancer patients who underwent ODG (ODG group). The mean age of the patients in the LADG group was 68.6 years, which was significantly higher than the mean age of 63.4 years in the ODG group. There were no statistically significant differences between the groups in operation time or preoperative risk score, but there were statistically significant differences in blood loss, surgical stress score, comprehensive risk score, and duration of postoperative hospital stay. We conclude that using the E-PASS scoring system, LADG appreciates a more beneficial procedure for the treatment of EGC than ODG.
\end{abstract}

Keywords: E-PASS; LADG; ODG

\section{Introduction}

In recent years laparoscopic surgery has become the main surgical treatment for early gastric cancer (EGC) [1-5], and the reasons have been standardization of the procedure, including lymph node dissection [6-8], reduced blood loss, and the rapid postoperative recovery associated with the reduction in size of the wound [5-7, 9]. Surgical stress greatly exceeding a patient's reserve capacity often disrupts the homeostasis of the respiratory, circulatory, metabolic, or immune systems, causing numerous postoperative complications. These postoperative complications may result from three major factors, namely, the quality of surgical performance, the patient's physiological status, and the degree of surgical stress applied. Where the quality of a surgical team has remained stable for a certain period, the morbidity and mortality rates

*Statement: The authors declare that they have no conflicts of interest.

\#Corresponding author. after an operation could be estimated by quantification of the patient's physiological status and the surgical stress. The Estimation of Physiologic Ability and Surgical Stress (E-PASS) was reported by Haga et al. [10]. This system comprises a preoperative risk score (PRS), a surgical stress score (SSS), and a comprehensive risk score (CRS) that is calculated from both the PRS and SSS. The Estimation of Physiologic Ability and Surgical Stress (EPASS) scoring system is used to evaluate surgical risk after elective digestive system surgery [10], and it predicts postoperative fatal complications [11-14]. Moreover, the E-PASS scoring system is useful for predicting and recognizing the risk of postoperative complications and for obtaining a better therapeutic outcome [15]. There are many reports about the value of general surgical risk in various surgical operations, but there were few reports concerning the comparison of LADG and ODG using E-PASS. We wanted to evaluate the feasibility of 
LADG for EGC by applying this system. We introduced laparoscopic surgery for the treatment of EGC in 2005. We therefore used the E-PASS scoring system to conduct a comparative study of the cases in which EGC treatment (open distal gastrectomy) was performed to treat stage IA gastric cancer during the 6-year period from 1999 to 2004 and the cases in which laparoscopy-assisted distal gastrectomy (LADG) was performed to treat stage IA gastric cancer cases during the period from 2005, when we introduced laparoscopic surgery, to 2010.

\section{Patients and Methods}

\subsection{Patients}

The subjects of this study were the patients who underwent surgical treatment for stage IA EGC at the Gastrointestinal Disease Center of Nippon Medical School Musasi Kosugi Hospital during the 12-year period from 1999 to 2010. Laparoscopic surgery was introduced in January 2005 and became the surgical treatment of first choice. The preoperative evaluations of depth of invasion and for the presence of lymph node metastasis were based on gastroscopy with gastric endoscopic ultrasonography, an upper gastrointestinal series, and an abdominal enhanced CT examination. All of the tumors were adenocarcinomas that had invaded either the mucosa or submucosa. Ultimately, 138 patients with stage IA gastric cancer were enrolled. Patients who had mucosal lesions that were suitable for endoscopic mucosal resection (lesion size $<20 \mathrm{~mm}$ when the elevated type and $<10$ $\mathrm{mm}$ when the depressed type) and patients who required combined surgery to treat another disease were excluded from the study. Patients who underwent ODG for stage IA EGC between 1999 and the end of 2004 were recruited as a control group. ODG was performed by the traditional procedure. A total of 69 patients were enrolled in the ODG group.

\subsection{Surgical Procedures}

LADG was performed by a 5-port technique with the patient under general anesthesia and in the supine position. A carbon dioxide pneumoperitoneum was created, and the camera was inserted through a $12-\mathrm{mm}$ subumbilical port. After the laparoscopic procedure, we made a single $5-\mathrm{cm}$ incision in the upper epigastric area, and the stomach, including the omentum and lymph nodes, was removed through the incision. Anastomosis was performed by a circular stapling technique, reconstructed by the Billroth-I, and a standard distal gastrectomy with a D1 + lymph node dissection was performed. Finally, the abdomen was closed in layers.

ODG plus D1 + lymph node dissection was performed through an upper midline incision and followed by Billroth-I reconstruction.

\subsection{Methods}

All clinical findings were collected retrospectively and were investigated. Nine factors determined the E-PASS scores, namely age, severity of heart disease, severity of pulmonary disease, diabetes mellitus, PS, American Society of Anesthesiologists physiological status classification, blood loss/body weight $(\mathrm{g} / \mathrm{kg})$, operation time (min), and extent of the skin incision. The equation of the E-PASS scoring system are shown in Table 1. The preoperative risk score (PRS) is used to evaluate preoperative risk, and it is calculated on the basis of age, whether severe heart disease is present, whether severe lung disease is present, diabetes, the American Society of Anesthesiologists (ASA) classification, and performance status index defined by the Japanese Society for Cancer Therapy (see Table 1 for the actual calculation method). The surgical stress score (SSS) is used to evaluate surgical risk, and it is calculated on the basis of the BMI, operation time, and blood loss (Table 1). The evaluation is not made based on these individual scores alone, but by an overall evaluation of the risk of surgery itself per-

Table 1. Statistical analysis of preoperative and surgical variables.

\begin{tabular}{cccc}
\hline Factors & LADG group & ODG group & $\mathrm{p}$ \\
\hline No. of subjects & 69 & 69 & \\
Sex M:F & $48: 21$ & $45: 24$ & 0.585 \\
Age (yr) & $68.6 \pm 9.9$ & $63.4 \pm 13.7$ & 0.040 \\
Operation time (min) & $237.5 \pm 68.0$ & $228.8 \pm 62.4$ & 0.594 \\
Blood loss (ml) & $162.1 \pm 369.9$ & $476.6 \pm 279.0$ & $<0.0001$ \\
Hospital stay (days) & $14.9 \pm 8.8$ & $23.6 \pm 6.7$ & $<0.0001$ \\
PRS & $0.359 \pm 0.189$ & $0.327 \pm 0.212$ & 0.037 \\
SSS & $-0.143 \pm 0.155$ & $0.275 \pm 0.093$ & $<0.0001$ \\
CRS & $-0.127 \pm 0.238$ & $0.247 \pm 0.208<0.0001$
\end{tabular}

*Formulas for calculating the Estimation of Physiologic Ability and Surgical Stress (E-PASS) scores: preoperative risk score (PRS), surgical stress score (SSS), and comprehensive risk score (CRS): 1) PRS $=-0.0686+0.00345 \mathrm{X} 1$ $+0.323 \mathrm{X} 2+0.205 \mathrm{X} 3+0.153 \mathrm{X} 4+0.148 \mathrm{X} 5+0.0666 \mathrm{X} 6 . \mathrm{X} 1$, age (yr); X2, presence (1) or absence ( 0 ) of severe heart disease; X3, presence (1) or absence $(0)$ of severe pulmonary disease; X4, presence (1) or absence (0) of diabetes mellitus; X5, performance status index (0 - 4); X6, American Society of Anesthesiologists physiological status classification $(1-5)$. Severe heart disease was defined as heart failure that was New York Heart Association Class III or IV, or severe arrhythmia requiring mechanical support. Severe pulmonary disease was defined as any condition with a $\% \mathrm{VC}$ below $60 \%$ and/or an FEV $1.0 \%$ below $50 \%$. Performance status index was based on the definition by the Japanese Society for Cancer Therapy. 2) SSS = $-0.342+0.0139 \mathrm{X} 1+0.0392 \mathrm{X} 2+0.352 \mathrm{X} 3$. X1, blood loss/body weight $(\mathrm{g} / \mathrm{kg})$; X2, operation time $(\mathrm{h}) ; \mathrm{X} 3$, extent of skin incision $(0$ : minor incisions for laparoscopic or thoracoscopic surgery (including scope-assisted surgery); a) laparotomy or thoracotomy alone; b) both laparotomy and thoracotomy). 3) $\mathrm{CRS}=-0.328+0.936(\mathrm{PRS})+0.976$ (SSS). VC, vital capacity; FEV, forced expiratory volume. 
formed on the basis of the PRS, the SSS, and the comprehensive risk score (CRS), which is calculated by adding the PRS and SSS together (Table 1). Postoperative complications were included only when medical or interventional treatment had been carried out. Complications included wound infection, anastomotic leakage, intraabdominal abscess, ileus, intra-abdominal bleeding, pneumonia, cardiac ischemic change, and organ dysfunction.

\subsection{Statistical Analysis}

All statistical analyses were performed using the JMP 9.0 statistical software program (SAS, Cary, NC, USA). P values less than 0.05 were considered statistically significant.

\section{Results}

Stage IA EGC was treated by LADG in 72 patients in our series, and by ODG in 69 patients. Three cases in the LADG group were excluded as mentioned below. Two of the three cases in the LADG group were excluded because they were ultimately diagnosed as stage IB, and the third case was excluded because it was ultimately diagnosed as stage II. The procedure in 4 other cases in the LADG group was converted to open laparotomy, because of severe adhesions in three cases and because of bleeding due to left liver lobe damage in the other case. Ultimately, 69 cases were enrolled in the LADG group. There were no complications in the LADG group. Mean age was 68.6 years in the LADG group and 63.4 years in the ODG group. The results of the statistical analysis showed no significant differences between the groups in sex or operation time (Table 1), but there were significant differences between them in age (Table 1), PRS (Table 1 and Figure 1), blood loss (Table 1), SSS (Table 1 and Figure 2), CRS (Table 1 and Figure 3), and length of postoperative hospital stay (Table 1).

\section{Discussion}

The E-PASS has been proposed as a means of predicting postoperative complications $[10,14,15]$. Recently, it is said that the E-PASS scoring system is useful for assessing the risk of surgical procedure and surgical decision making [16-18]. Laparoscopic surgery has been widely adopted to treat EGC in recent years [1,2], and its advantages have been pointed out by many papers [3-5]. The greatest benefit it has provided to patients is the speed of postoperative recovery, the main reason being the small size of the surgical wound. The advantages of laparoscopic surgery have certainly been said to lie in the small size of the surgical wound and the small volume of blood loss, and there was significantly less blood loss in the LADG group according to the results of the present study

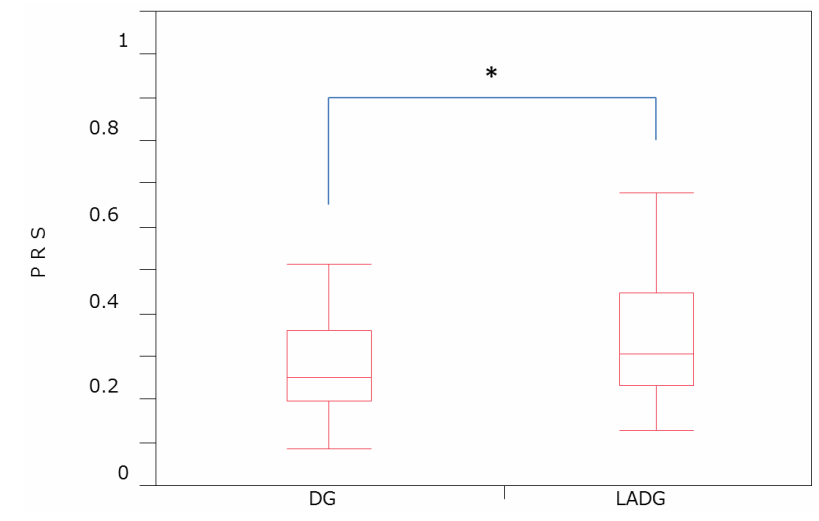

Figure 1. There was a significant difference in PRS between the ODG group and LADG group. The quantile box plot is shown in the form of a red line. ${ }^{*} \mathbf{p}=\mathbf{0 . 0 3 7}$.

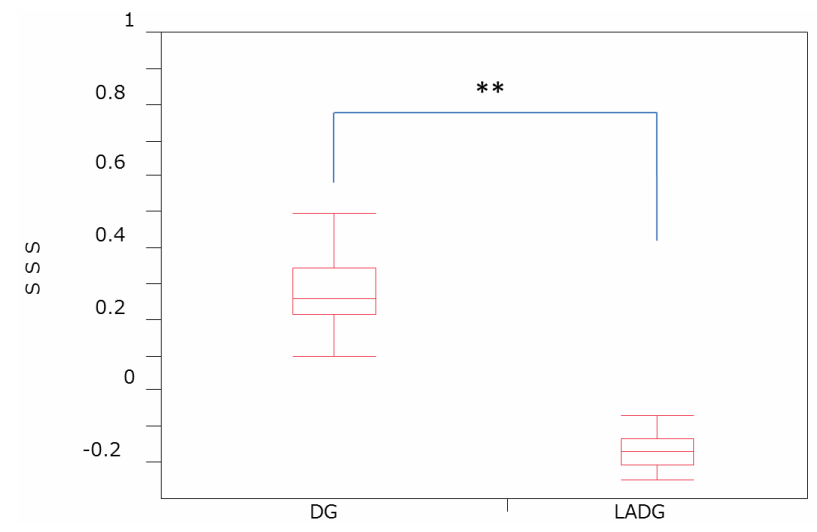

Figure 2. There was a significant difference in SSS between the ODG group and LADG group. ${ }^{* *} \mathrm{p}<\mathbf{0 . 0 0 0 1}$.

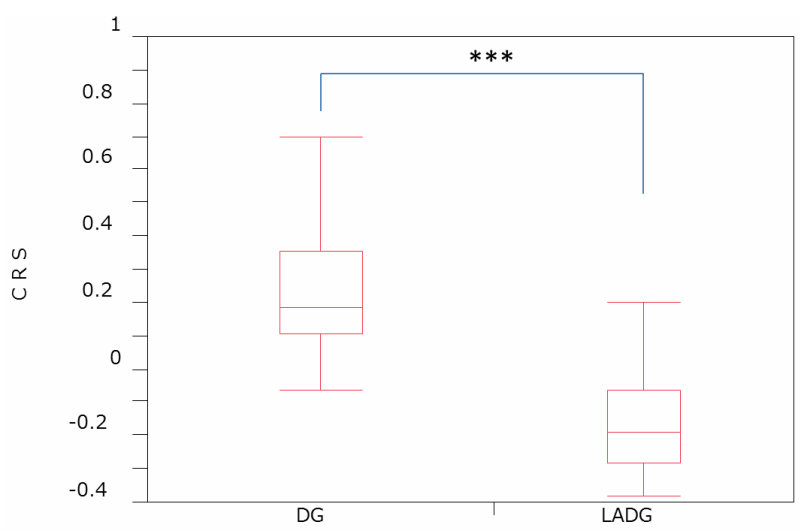

Figure 3. There was a significant difference in CRS between the ODG group and LADG group. ${ }^{* * *} \mathrm{p}<\mathbf{0 . 0 0 0 1}$.

as well. However, the drawback of laparoscopic surgery in the form of the long operation time has become a problem. We thought that this problem could be overcome by striving to standardize the surgical procedure, establish a regular surgical team, and introduce appropriate surgical instruments, and, actually, no significant 
difference in operation time was observed in this study, and as a result the SSS was significantly lower in the LADG group. On the other hand, surgical risk cannot be concluded to be decreased just because the PRS is lower, because in elderly patients and patients who have highrisk complications the operation sometimes ends in palliative surgery rather than seeking curative surgery [1618]. In our present study mean age was higher in the LADG group, and the PRS was higher. This means that the preoperative risk was higher in the LADG group, but since the aforementioned SSS was lower, the overall evaluation in the form of the CRS was significantly lower in the LADG group. This also seems to suggest that laparoscopic surgery is more beneficial as a treatment for EGC, even if there is some risk. A CRS of 1.0 is said to be the threshold score for postoperative fatal complications increasing [10]. In the present study the CRS was significantly lower in the LADG group, but neither score exceeded the threshold. Moreover, the postoperative hospital stay was also significantly shorter in the LADG group. Laparoscopic surgery has been described as more beneficial in many papers [1-4], but few of them have reported a comparative study in which the E-PASS was used. In the present study we used the E-PASS to perform an overall evaluation of the surgical treatment for EGC, and based on the results we concluded that surgical treatment in the LADG group was more beneficial. This corroborates what has been widely claimed, and it is a result that it was obtained according to an overall evaluation of the patients' risk and the risk of surgery itself, a different viewpoint from the past. Based on the above, the E-PASS scoring system is useful for assessing the risks of the operation procedure of EGC. We appreciate that LADG is more feasible than ODG as a surgical treatment for EGC.

\section{REFERENCES}

[1] S. Kitano, N. Shiraishi, I. Uyama, K. Sugihara, N. Tanigawa and Japanese Laparoscopic Surgery Study Group, "A Multicenter Study on Oncologic Outcome of Laparoscopic Gastrectomy for Early Cancer in Japan," Annals of Surgery, Vol. 245, No. 1, 2007, pp. 68-72. http://dx.doi.org/10.1097/01.sla.0000225364.03133.f8

[2] H. Yano, et al., "The Usefulness of Laparoscopy-Assisted Distal Gastrectomy in Comparison with That of Open Distal Gastrectomy for Early Gastric Cancer," Gastric Cancer, Vol. 4, No. 2, 2001, pp. 93-97. http://dx.doi.org/10.1007/PL00011730

[3] C. G. Huscher, et al., "Laparoscopic versus Open Subtotal Gastrectomy for Distal Gastric Cancer: Five-Year Results of a Randomized Prospective Trial," Annals of Surgery, Vol. 241, No. 2, 2001, pp. 232-237.

http://dx.doi.org/10.1097/01.sla.0000151892.35922.f2

[4] S. Kitano, et al., "A Randomized Controlled Trial Com- paring Open vs Laparoscopy-Assisted Distal Gastrectomy for the Treatment of Early Gastric Cancer: An Interim Report," Surgery, Vol. 131, Supplement 1, 2002, pp. S306S311. http://dx.doi.org/10.1067/msy.2002.120115

[5] K. J. Weber, Reyes CD, Gagner M, Divino CM, "Comparison of Laparoscopic and Open Gastrectomy for Malignant Disease," Surgical Endoscopy, Vol. 17, No. 6, 2003, pp. 968-971.

http://dx.doi.org/10.1007/s00464-002-8738-5

[6] K. Yasuda, et al., "Rate of Detection of Lymph Node Metastasis Is Correlated with the Depth of Submucosal Invasion in Early Stage Gastric Carcinoma," Cancer, Vol. 85, No. 10, 1999, pp. 2119-2123.

http://dx.doi.org/10.1002/(SICI)1097-0142(19990515)85: 10<2119::AID-CNCR4>3.0.CO;2-M

[7] S. Miura, et al., "Laparoscopy-Assisted Distal Gastrectomy with Systemic Lymph Node Dissection: A Critical Reappraisal from the Viewpoint of Lymph Node Retrieval," Journal of the American College of Surgeons, Vol. 198, No. 6, 2004, pp. 933-938. http://dx.doi.org/10.1016/j.jamcollsurg.2004.01.021

[8] M. Fujiwara, et al., "Laparoscopy-Assisted Distal Gastrectomy with Systemic Lymph Node Dissection for Early Gastric Carcinoma: A Review of 43 Cases," Journal of the American College of Surgeons Vol. 196, No. 1, 2003 , pp. $75-81$.

http://dx.doi.org/10.1016/S1072-7515(02)01539-9

[9] K. Fujii, et al., "T Lymphocyte Subsets and Th1/Th2 Balance after Laparoscopy-Assisted Distal Gastrectomy," Surg Endosc, Vol. 17, No. 9, 2003, pp. 1440-1444. http://dx.doi.org/10.1007/s00464-002-9149-3

[10] Y. Haga, et al., "Estimation of Physiologic Ability and Surgical Stress (E-PASS) for a Surgical Audit in Elective Digestive Surgery," Surgery, Vol. 135, No. 6, 2004, pp. 586-594. http://dx.doi.org/10.1016/j.surg.2003.11.012

[11] D. Hashimoto, et al., "Can the Physiologic Ability and Surgical Stress (E-PASS) Scoring System Predict Operative Morbidity after Distal Pancreatectomy?" Surgery Today, Vol. 40, No. 7, 2004, pp. 632-637. http://dx.doi.org/10.1007/s00595-009-4112-8

[12] Y. Haga, K. Ikejiri, H. Takeuchi, M. Ikenaga and Y. Wada, "Value of General Surgical Risk Models for Predicting Postoperative Liver Failure and Mortality Following Liver Surgery," Journal of Surgical Oncology, Vol. 106, No. 7, 2012, pp. 898-904. http://dx.doi.org/10.1002/jso. 23160

[13] A. Kotera, Y. Haga, J. Kei, M. Okamoto and K. Seo, "Evaluation of Estimation of Physiologic Ability and Surgical Stress to Predict In-Hospital Mortality in Cardiac Surgery," Journal of Anesthesia, Vol. 25, No. 4, 2011, pp. 481-491. http://dx.doi.org/10.1007/s00540-011-1162-z

[14] V. M. Banz, P. Studer, D. Inderbitzin and D. Candinas, "Validation of the Estimation of Physiologic Ability and Surgical Stress (E-PASS) Score in Liver Surgery," World Journal of Surgery, Vol. 33, No. 6, 2009, pp. 1259-1265. http://dx.doi.org/10.1007/s00268-009-9989-2

[15] Y. Oka, et al., "Usefulness of an Estimation of Physiologic Ability and Surgical Stress (E-PASS) Scoring Sys- 
tem to Predict the Incidence of Postoperative Complications in Gastrointestinal Surgery," World Journal of Surgery, Vol. 29, No. 8, 2005, pp. 1029-1033. http://dx.doi.org/10.1007/s00268-005-7719-y

[16] T. Tang, et al., "Estimation of Physiologic Ability and Surgical Stress (E-PASS) as a Predictor of Immediate Outcome after Elective Abdominal Aortic Aneurysm Surgery," The American Journal of Surgery, Vol. 194, No. 2, 2007, pp. 176-182.

http://dx.doi.org/10.1016/j.amjsurg.2006.10.032

[17] J. Hirose, H. Mizuta, J. Ide and K. Nomura, "Evaluation of Estimation of Physiologic Ability and Surgical Stress (E-PASS) to Predict the Postoperative Risk for Hip Fracture in Elder Patients," Archives of Orthopaedic and Trauma Surgery, Vol. 128, No. 12, 2008, pp. 1447-1452. http://dx.doi.org/10.1007/s00402-007-0551-0

[18] K. Koushi, et al., "Using the E-PASS Scoring System to Estimate the Risk of Emergency Abdominal Surgery in Patients with Acute Gastrointestinal Disease," Surg Today, Vol. 41, No. 11, 2011, pp. 1481-1485. http://dx.doi.org/10.1007/s00595-010-4538-z 